- 研究报告・

\title{
天山雪岭云杉森林群落的密度制约效应
}

\author{
王慧杰 ${ }^{1}$ 常顺利 ${ }^{*} \quad$ 张毓涛 $^{2}$ 谢 锦 $^{1}$ 何 平 $^{1}$ 宋成程 ${ }^{1}$ 孙雪娇 $^{1}$ \\ 1 (新疆大学资源与环境科学学院绿洲生态教育部重点实验室, 乌鲁木齐 830046) \\ 2 (新疆林业科学院森林生态研究所, 乌鲁木齐 830063)
}

\begin{abstract}
摘要: 密度制约是维持自然森林群落物种共存的重要机制之一。雪岭云杉(Picea schrenkiana) 是天山森林群落的单 优种, 在我国温带森林中占有重要地位。本文基于对天山雪岭云杉 8 ha森林动态监测样地的两次调查结果, 分析了 该样地群落物种组成的动态变化, 运用点格局方法分析了雪岭云杉个体在不同龄级的空间分布格局及其关联性, 探讨了该群落的密度制约效应。结果表明: (1)该样地内, DBH $\geqslant 1 \mathrm{~cm}$ 的木本植物在2009年有11,835株, 2014年为 11,050 株, 5 年间的个体死亡率为 $8.82 \%$, 补员率为 $2.19 \%$, 个体株数净减少 $6.63 \%$ 。(2)使用双关联函数 $g(\mathrm{r})$ 分析了不 同龄级雪岭云杉的空间分布格局, 发现幻龄树和中龄树在 0-40 m尺度上主要呈现聚集分布，但随着尺度增大聚集 强度逐渐减小; 老龄树在0-2 m及 $4 \mathrm{~m}$ 尺度上呈随机分布, 在其他尺度上呈聚集分布, 但聚集程度较小。(3)用“案例 一对照”设计的方法，排除生境异质性的影响后，将幼龄树和中龄树作为案例，老龄树作为对照，对比幼龄树、中龄 树与老龄树的分布格局, 发现雪岭云杉幼龄树和中龄树在 0-40 m尺度上呈现额外的聚集, 随着径级的增大, 这种 额外的聚集强度逐渐减小, 即表现出密度制约效应。(4)老龄树与中龄树、幼龄树的空间关联性相同, 在0-40 m尺 度上均呈明显负关联。
\end{abstract}

关键词: 动态监测样地；密度制约假说；空间分布；雪岭云杉；天山

\section{Density-dependent effects in Picea schrenkiana forests in Tianshan Mountains}

\author{
Huijie Wang ${ }^{1}$, Shunli Chang ${ }^{1 *}$, Yutao Zhang ${ }^{2}$, Jin Xie ${ }^{1}$, Ping $\mathrm{He}^{1}$, Chengcheng Song ${ }^{1}$, Xuejiao Sun ${ }^{1}$ \\ 1 Key Laboratory of Oasis Ecology of Ministry of Education, College of Resource and Environment Sciences, Xinjiang \\ University, Urumqi 830046 \\ 2 Institute of Forest Ecology, Xinjiang Academy of Forestry, Urumqi 830063
}

\begin{abstract}
The density-dependence hypothesis is one of the most important mechanisms proposed to explain species coexistence in natural forest communities. Picea schrenkiana is the single dominant species of the forest communities in Tianshan Mountains, which plays a very important role in temperate forests of China. We used reexamined data with a five-year interval on an 8-ha $P$. schrenkiana forest dynamics plot to illustrate the dynamics of species composition. Pointpattern analysis was conducted to reveal the spatial structure of $P$. schrenkiana individuals of different ages, and the effects of the density-dependence hypothesis on different ages of $P$. schrenkiana was discussed. The results were as follows: (1) Woody plants with DBH $\geqslant 1 \mathrm{~cm}$ were 11,835 trees in 2009 and 11,050 in 2014 . The mortality rate was $8.82 \%$, the recruitment rate was $2.19 \%$, and the net decrease was $6.63 \%$; (2) Young and medium trees of P. schrenkiana presented an aggregation distribution across a scale of $0-40 \mathrm{~m}$, and the aggregation intensity decreased as the scale increased. Mature trees presented a random distribution on scales of $0 \mathrm{~m} \leqslant \mathrm{r} \leqslant 2 \mathrm{~m}$ and $\mathrm{r}=4 \mathrm{~m}$, and an aggregation distribution on other scales with a low density. (3) Using a "case-control" design method and eliminating habitat heterogeneity, the distribution of young and medium trees were taken as examples of case. The distribution pattern of mature trees was used as a comparison and represented habitat heterogeneity, and was compared with that of young and medium trees. Young and medium trees of $P$. schrenkiana were observed to present
\end{abstract}

收稿日期: 2015-10-21; 接受日期: 2016-01-11

基金项目: 国家自然科学基金(U1503187)和新疆自治区科技支撑计划项目(201331125)

* 通讯作者 Author for correspondence. E-mail: ecocsl@163.com 
more considerable gathering than was observed for mature trees on scales of 0-40 m, and density decreased while the diameter class increased. These results indicate that the density-dependence hypothesis has an influence on distribution patterns of different age classes. This study reveals that $P$. schrenkiana was affected by habitat heterogeneity and presented a significant aggregation effect. The spatial pattern of $P$. schrenkiana is revealed to be affected by the density-dependence hypothesis after eliminating the effects of habitat heterogeneity. The results of this paper support the Janzen-Connell hypothesis.

Key words: dynamics plot; density-dependence hypothesis; spatial distribution; Picea schrenkiana; Tianshan Mountains

在大型固定样地平台上研究群落内物种多样 性的维持机制是当前生态学研究中最活跃的领域 之一(Hubbell et al, 2001; Wang et al, 2011; 马克平, 2013), 而密度制约被认为是解释群落物种多样性 维持的重要机制之一。密度制约是发生在同种邻株 之间的调节机制，它通过增加同种邻株的死亡率， 加大树木个体的间距; 如果密度制约起作用, 那么 随着时间的推移或径级的增加, 种群的聚集程度应 该呈现出下降趋势。密度制约假说认为, 资源竞争、 有害生物侵害和化感作用等导致同种物种之间相 互损害，从而为其他物种的生存提供了空间和资 源, 促进了物种共存(Janzen et al, 1970; Connell et al，1971; 祝燕等，2009)。40多年来，该理论受到广 泛关注和讨论(Stoll \& Newbery, 2005; Chen et al, 2010; Zhu et al, 2010; Piao et al, 2013), 众多的野外 观测和实验研究认为密度制约是普遍存在的 (Gunton \& Kunin, 2009; Zhang et al, 2009; Bagchi et al, 2011)。然而一些学者认为, 密度制约的普遍性及 其强度可能受其他因素干扰, 如研究阶段、地形、 分析方法等, 从而在某些研究区并不常见(骆争荣, 2011; Luo et al, 2012; Guo et al, 2015)。目前，对该理 论的验证和研究仍然存在许多值得探讨的问题, 例 如，密度制约在单一森林类型中是否存在？如果存 在，它在时空尺度上呈现的效应如何?

一般认为，密度制约效应主要发生在物种生活 史的早期阶段, 如种子和幼苗阶段, 但这个时期容 易受到专一性天敌的侵害而表现出较高的死亡率, 从而影响密度制约的检验结果(Luo et al, 2012; 王 薇等, 2014; 郭屹立等, 2015)。众多研究表明, 除了 通过研究幼苗的死亡率来分析密度制约效应之外, 通过研究物种不同生长阶段的空间格局变化趋势 来推断密度制约效应也是一种可行的方法(Condit et al, 2000; Getzin et al, 2008; 王婷等, 2014)。
雪岭云杉(Picea schrenkiana) 是天山森林的单 优树种, 其分布格局对天山森林群落结构的构成具 有重要作用。研究雪岭云杉不同生长阶段个体的空 间分布规律及其关联性和密度制约效应, 有助于掌 握该物种在天山地区的生长现状和其群落的发展 趋势, 从而为探讨雪岭云杉森林植物物种多样性及 其维持机制提供帮助，对实现其有效保护和可持续 利用具有重要意义。

本文以天山雪岭云杉 8 ha森林动态监测样地为 对象, 在 2009年和2014年两次样地调查数据的基础 上, 对群落内雪岭云杉个体组成动态和物种密度制 约效应进行了分析，试图回答以下两个问题：(1)天 山雪岭云杉森林内木本植物死亡和补员的短期动 态情况如何; (2)密度制约效应对天山雪岭云杉林群 落的维持是否起到主要作用。

\section{1 方法}

\section{1 样地概况}

天山雪岭云杉 8 ha森林动态监测样地 $\left(43^{\circ} 25^{\prime}-\right.$ $43^{\circ} 26^{\prime} \mathrm{N}, 8^{\circ} 27^{\prime}-87^{\circ} 29^{\prime} \mathrm{E}$ )位于天山北坡中部乌鲁木 齐县板房沟林场, 依托于中国森林生态系统研究网 络(CFERN)天山森林生态系统定位研究站(图1)。研 究区属温带大陆性气候, 年总辐射量达 $5.85 \times 10^{5}$ $\mathrm{J} /\left(\mathrm{cm}^{2}\right)$, 年均气温约为 $2-3^{\circ} \mathrm{C}$, 历年极端最高温为 $30.5^{\circ} \mathrm{C}$, 极端最低温为 $-30.2^{\circ} \mathrm{C}$; 年降水量 400-600 $\mathrm{mm}$, 最大积雪深度为 $65 \mathrm{~cm}$ (阿米娜木. 艾力等, 2014)。

天山林区以雪岭云杉纯林为主, 林缘、林窗及 林下的乔木主要有密叶杨(Populus talassica)和天山 花楸(Sorbus tianschanica)、天山桦(Betula tianschanica)等; 灌木主要有栒子(Cotoneaster melanocarpus)、小檗(Berberis heteropoda)、蔷薇(Rosa spinosissima)、绣线菊(Spiraea hypericifolia)、方枝 


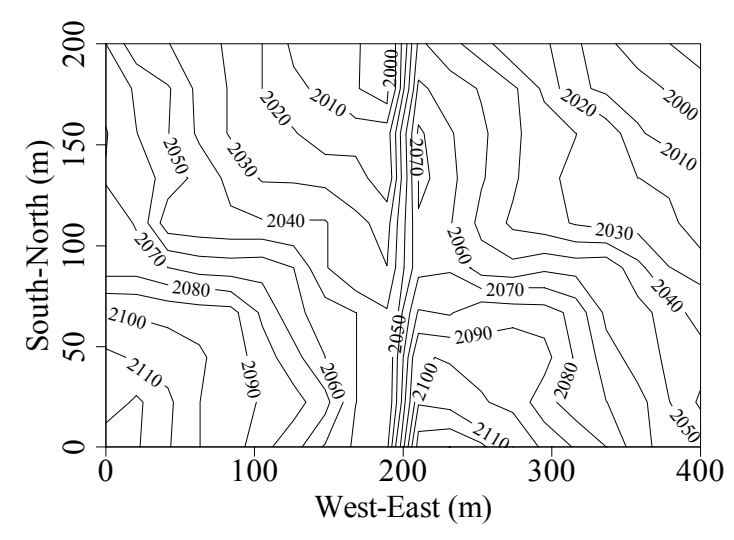

图1 天山雪岭云杉8 ha森林动态监测样地示意图

Fig. 1 The sketch map of the 8-ha Picea schrenkiana forest dynamics plot in Tianshan Mountains

柏 (Juniperus pseudosabina)、锦鸡儿 (Caragana leucophloea)和忍冬(Lonicera hispida)等, 林下草本 植物主要有老鹳草(Geranium rotundifolium)、羽衣草 (Alchemilla tianschanica) 和羊角芹(Aegopodium podagraria)等; 林下土壤均为山地灰褐色森林土(仇 瑶等, 2015)。

\section{2 样地建立}

依据CTFS (Center for Tropical Forest Science) 样地建设技术规范(Condit, 1998), 用全站仪将8 ha 样地划分为 200 个 $20 \mathrm{~m} \times 20 \mathrm{~m}$ 的样方, 样方的 4 个角 用水泥桩作永久标记。2009年7-9月, 对样地进行了 首次调查(张毓涛等, 2011a, b), 主要内容包括: 鉴 定样地内所有胸径 $(\mathrm{DBH}) \geqslant 1 \mathrm{~cm}$ 的木本植物种 类, 测量其胸径、树高、坐标, 记录状态(存活、倒 伏、枯立), 并对其定位挂牌, 测量每个小样方的地 形数据(海拔、坡度、凹凸度)和样地基本状况(郁闭 度和草本盖度)。2014年7-9月进行了复查。

\section{3 数据分析}

\subsection{1 物种重要值的计算方法}

物种重要值是群落内物种优势度的重要度量 标志(王伯荪, 1987), 可用以判断群落内各物种的相 对重要性及适应性, 其计算公式如下 (张金屯, 1995):

重要值 (importance value, IV) $=($ 相对密度 + 相 对频度+相对优势度) $/ 3$

\subsection{2 龄级的划分}

在相同的环境条件下, 同一物种的径级和龄级 对环境的适应性具有一致性(李立等, 2010)。本文结
合本样地实际情况，利用径级代替龄级的方法 (Bagchi et al, 2011), 对雪岭云杉个体大小进行划 分。具体操作如下: 将雪岭云杉的龄级以样地所有 同种个体径级的 $99 \%$ 分位数(DBH99)来划分, 其中 $\mathrm{DBH} \leqslant \mathrm{DBH} 99^{1 / 3}$ 定义为幼龄树, $\mathrm{DBH} 99^{1 / 3}<\mathrm{DBH}$ $\leqslant \mathrm{DBH} 99^{3 / 5}$ 定义为中龄树, $\mathrm{DBH}>\mathrm{DBH} 99^{3 / 5}$ 定义 为老龄树。因此, 本文定义的雪岭云杉幼龄树的径 级为 $1 \mathrm{~cm} \leqslant \mathrm{DBH} \leqslant 12.5 \mathrm{~cm}$, 中龄树为 $12.5 \mathrm{~cm}$ $<\mathrm{DBH} \leqslant 22.5 \mathrm{~cm}$, 老龄树为DBH $>22.5 \mathrm{~cm}$ 。

\subsection{3 点格局分析}

采用现代点格局函数分析各径级的空间分布 特征和不同生活史阶段之间的空间关联。Ripley's $K$ 函数公式如下(Ripley, 1976):

$$
K(\mathrm{r})=\frac{A}{\mathrm{n}^{2}} \sum_{\mathrm{i}=1}^{n_{1}} \sum_{\mathrm{j}=1}^{n_{2}} \frac{I_{\mathrm{r}}\left(d_{\mathrm{ij}}\right)}{W_{\mathrm{ij}}}(\mathrm{i} \neq \mathrm{j})
$$

其中, $A$ 为研究样方的面积, $n$ 为样地内物种的总个 体数, $r$ 为以目标树为圆点的取样圆半径, $I_{\mathrm{r}}\left(d_{\mathrm{ij}}\right)$ 为指 示函数, $d_{\mathrm{ij}}$ 为圆心点 $i$ 和点 $j$ 之间的距离。当 $d_{\mathrm{ij}} \leqslant r$ 时, $I_{\mathrm{r}}\left(d_{\mathrm{ij}}\right)=1$, 当 $d_{\mathrm{ij}}>r$ 时, $I_{\mathrm{r}}\left(d_{\mathrm{ij}}\right)=0 ; W_{\mathrm{ij}}$ 为权重值。

双变量的 $K$ 函数公式为:

$$
K_{12}(\mathrm{r})=\frac{A}{n_{1} n_{2}} \sum_{\mathrm{i}=1}^{n_{1}} \sum_{\mathrm{j}=1}^{n_{2}} \frac{I_{\mathrm{r}}\left(d_{\mathrm{ij}}\right)}{W_{\mathrm{ij}}}(\mathrm{i} \neq \mathrm{j})
$$

式中, $n_{1}$ 与 $n_{2}$ 表示不同生活史阶段的个体数量。

成对相关函数(简称 $g$ 函数)消除了 $K$ 函数在大尺 度上的累积效应, 与Ripley's $K$ 函数相比, $g(\mathrm{r})$ 能较 敏感地判别某一尺度上点的实际分布偏离期望值 的程度, 是评估聚集程度的重要分析方法。g函数有 相应的单变量和双变量形式, 其表达式分别为:

$$
\begin{aligned}
& g(\mathrm{r})=\frac{d K(\mathrm{r})}{2 \pi r d r} \\
& g_{12}(\mathrm{r})=\frac{d K_{12}(\mathrm{r})}{2 \pi r d r}
\end{aligned}
$$

$g(\mathrm{r})$ 值的大小代表了聚集强度的大小, 当 $g(\mathrm{r})=$ 1 时为随机分布; 当 $g(r)>1$ 时为聚集分布; 当 $g(r)<$ 1 时为均匀分布。

\subsection{4 零模型的选择}

选择具有明确生态学意义、能准确描述数据偏 离理论程度的零假设模型(null model)对空间点格 局分析非常关键。不同的研究目的和问题假设需要 选择不同的零模型(王金锤厅等, 2012)。本文运用单变 
量的Ripley's $K$ 函数和 $g(\mathrm{r})$ 函数分析物种空间格局; 用双变量的Ripley's $K$ 函数和 $g(\mathrm{r})$ 函数分析不同径级 间的空间关联性, 用到的零模型有: (1)完全空间随 机模型(complete spatial random-ness, CSR): 假设物 种的空间分布不受任何生物或非生物过程影响, 在 研究区域内各点出现的概率相同; (2)随机标签模型 (random labeling, RL): 个体的位置不变, 属性随机 赋予每个个体。为了提高种群空间分布格局的分析 精度, 本文设定栅格大小为 $1 \mathrm{~m} \times 1 \mathrm{~m}$, 圆环宽度为 1 $\mathrm{m}$, 尺度域为 $0-40 \mathrm{~m}$ 。以上两种零模型均利用 199 次 Monte Carlo随机模拟, 分别利用模拟的最大值和最 小值生成上下两条包迹线，计算 $99 \%$ 的置信区间。

\subsection{5 密度制约效应分析方法}

使用随机标签模型中的案例-控制设计法来检 验在植物不同生长阶段密度制约效应对种群的调 节作用，使用案例－对照设计来说明生境异质性对 种群的影响(Diggle \& Chetwynd, 1991)。由于导致生 境异质性效应的因子难以用数值指标量化, 故假定 生境异质性对树木的影响在不同生长阶段都是一 直存在的, 到了老龄树时期, 生境异质性对种群分 布格局的调控基本稳定, 这样老龄树的分布格局就 显示了生境异质性作用的结果(Zhu et al, 2010)。因 此, 用老龄树在样地中的分布格局作为对照(格局1) 来代表生境异质性因子, 幼龄树和中龄树作为案例 格局(格局2), 通过案例与对照的比较, 排除生境异 质性的作用来研究个体之间真实的相互作用, 从而 推断其群落过程。如 $g_{21}$ 表示案例周围对照个体的分 布强度, $g_{22}$ 表示案例周围案例个体的分布强度, 以 此类推。

若 $g_{21}(r)-g_{22}(r) \approx 0$, 则实际分布的值落在包迹 线内, 符合零模型, 案例和对照组间的分布格局没 有差异; 若 $g_{21}(\mathrm{r})-g_{22}(\mathrm{r})<0$, 则表明作为案例的中 龄树和幼树树比老龄树更加聚集(Getzin et al, 2008)。从幼龄树到中龄树聚集格局的降低, 表示存 在密度制约效应, 聚集格局降低的程度指示密度制 约效应的强度。用公式(6)来检验密度制约的统计 分析:

$$
d(\mathrm{r})=d \mathrm{j}(\mathrm{r})-d \mathrm{~s}(\mathrm{r})
$$

其中 $d \mathrm{j}(\mathrm{r})$ 为中龄树作为案例时 $g_{21}(\mathrm{r})-g_{22}(\mathrm{r})$ 的差值, $d \mathrm{~s}(r)$ 为幼龄树作为案例时 $g_{21}(\mathrm{r})-g_{22}(\mathrm{r})$ 的差值。若 $d(r)>0$, 说明存在密度制约效应。
空间点格局分析要求样地中独立个体数 $\geqslant 30$, 并且每个物种每个径级的平均个体数都要 $\geqslant 30$, 而在该 8 ha样地内仅雪岭云杉一个物种符合此条 件。因此, 本文分别用2009年和2014年的调查数据 对雪岭云杉不同龄级在 $40 \mathrm{~m}$ 尺度范围内的空间分 布格局进行分析。将分析结果对比后发现, 2009年 和2014年的结果并无显著差异, 因此, 本文仅列出 了2014年调查数据的分析结果。

\subsection{6 数据分析与制图}

采用空间格局分析软件Programita (Wiegand \& Moloney，2004) 和 R 软 件 (R 3.1.2; http://www.rroject.org/)进行数据分析和绘图。

\section{2 结果}

\section{1 样地物种组成及动态变化}

在 2009 年, 样地中 $\mathrm{DBH} \geqslant 1$ 的存活个体共有 11,835 株, 分属于 6 科 8 属 8 种, 其中乔木 4 种, 灌木 4 种。到2014年, 样地中DBH $\geqslant 1$ 的存活个体共有 11,050 株，分属于 5 科 6 属 6 种，其中乔木 3 种，灌木 3 种, 天山桦和栒子因个体全部死亡而在本样地内消 失(表 1$)$ 。两次调查中重要值 $\geqslant 1 \%$ 的物种有 4 种, 分 别是雪岭云杉、小檗、天山花楸和忍冬。稀有种(每 公顷个体数量不多于 1 株)有 4 种, 分别是密叶杨、天 山桦、栒子和蓄薇。5年间 $\mathrm{DBH} \geqslant 1 \mathrm{~cm}$ 的独立个 体数减少了 785 株(减少幅度 $6.63 \%$ ), 其中死亡个体 为 1,044 株 (死亡率 $8.82 \%$ ), 补员个体为 259 株 (补员 率 $2.19 \%$ ); 补员和死亡个体数最多的物种为雪岭云 杉, 这与其重要值最大有关。

2009年样地内所有调查个体平均胸径和平均 树高分别为 $13.10 \mathrm{~cm}$ 和 $10.39 \mathrm{~m}, 2014$ 年分别为 14.18 $\mathrm{cm}$ 和 $11.21 \mathrm{~m}, 5$ 年间所有调查个体平均胸径和平均 树高分别增长了 $1.08 \mathrm{~cm}$ 和 $0.82 \mathrm{~m}$ 。

\section{2 雪岭云杉的空间分布格局}

天山雪岭云杉 3 个齢级的个体均遍布整个样地 (图2)。2014年, 幼龄树、中龄树和老龄树的个体数 分别为5,667株、 3,189 株和 2,106 株, 各占该样地雪 岭云杉总株数的 $51.70 \%, 29.09 \%$ 和 $19.21 \%$ 。幼龄树 和中龄树均呈现出明显的聚集分布, 老龄树聚集程 度相对较小。

完全随机分布模型结果表明, 幼龄树和中龄树 均在0-40 m尺度上呈现聚集分布, 随着尺度的 
表1 2009和2014年天山雪岭云杉 8 ha森林动态监测样地的群落动态

Table 1 Community dynamics in the 8-ha Picea schrenkiana forest dynamics plot in Tianshan Mountains in 2009 and 2014

\begin{tabular}{|c|c|c|c|c|c|c|c|c|c|c|}
\hline \multirow[t]{2}{*}{$\begin{array}{l}\text { 物种 } \\
\text { Species }\end{array}$} & \multirow[t]{2}{*}{$\begin{array}{l}\text { 生长型 } \\
\text { Growth form }\end{array}$} & \multicolumn{2}{|c|}{$\begin{array}{l}\text { 多度 } \\
\text { Abundance }\end{array}$} & \multicolumn{2}{|c|}{$\begin{array}{l}\text { 重要值 } \\
\text { Importance value }(\%)\end{array}$} & \multicolumn{2}{|c|}{$\begin{array}{l}\text { 平均胸径 } \\
\text { Mean DBH }(\mathrm{cm})\end{array}$} & \multirow{2}{*}{$\begin{array}{l}\text { 死亡数 } \\
\text { Number of } \\
\text { dead } \\
\text { individual }\end{array}$} & \multirow{2}{*}{$\begin{array}{l}\text { 补员数 } \\
\text { Number of } \\
\text { recruited } \\
\text { individual }\end{array}$} & \multirow{2}{*}{$\begin{array}{l}\text { 胸径净增长 } \\
\text { DBH net } \\
\text { growth }(\mathrm{cm})\end{array}$} \\
\hline & & 2009 & 2014 & 2009 & 2014 & 2009 & 2014 & & & \\
\hline 雪岭云杉 Picea schrenkiana & 乔木 Tree & 11,731 & 10,962 & 92.88 & 92.51 & 13.16 & 14.25 & 1,010 & 241 & 1.03 \\
\hline 密叶杨 Populus talassica & 乔木 Tree & 5 & 5 & 0.42 & 0.41 & 9.20 & 10.88 & 0 & 0 & 1.68 \\
\hline 天山桦 Betula tianschanica & 乔木 Tree & 1 & 0 & 0.14 & 0.00 & 4.90 & 5.20 & 1 & 0 & - \\
\hline 天山花楸 Sorbus tianschanica & 乔木 Tree & 31 & 25 & 1.45 & 2.58 & 8.31 & 7.81 & 8 & 2 & 0.68 \\
\hline 小檗 Berberis heteropoda & 灌木 Shrub & 34 & 40 & 2.22 & 2.74 & 2.11 & 2.05 & 8 & 14 & 0.16 \\
\hline 忍冬 Lonicera hispida & 灌木 Shrub & 27 & 17 & 2.08 & 1.63 & 4.91 & 5.94 & 11 & 1 & 0.80 \\
\hline 栒子 Cotoneaster melanocarpus & 灌木 Shrub & 4 & 0 & 0.54 & 0.00 & 5.56 & 0.00 & 4 & 0 & - \\
\hline 蓄薇 Rosa spinosissima & 灌木 Shrub & 2 & 1 & 0.27 & 0.13 & 4.60 & 3.30 & 2 & 1 & - \\
\hline 合计 Total & & 11,835 & 11,050 & 100 & 100 & & & 1,044 & 259 & \\
\hline
\end{tabular}

A

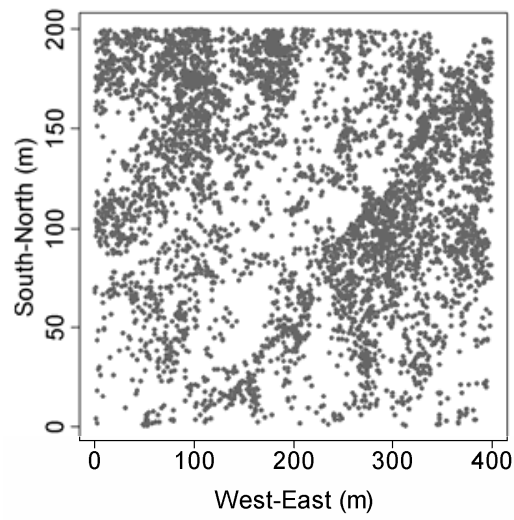

B

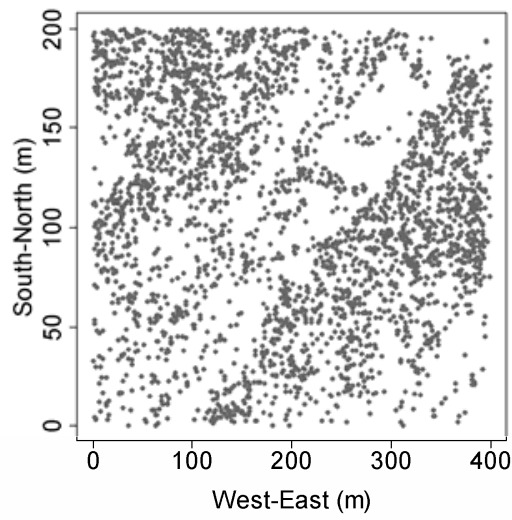

C

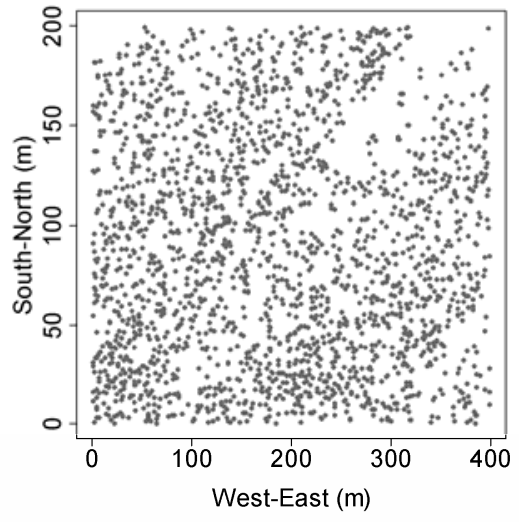

图2 天山雪岭云杉 $8 \mathrm{ha}$ 森林动态监测样地内不同龄级(径级)雪岭云杉的空间分布格局。(A)幼龄树 $(1 \mathrm{~cm} \leqslant \mathrm{DBH} \leqslant 12.5$ $\mathrm{cm})$; (B)中龄树 $(12.5 \mathrm{~cm}<\mathrm{DBH} \leqslant 22.5 \mathrm{~cm})$; (C)老龄树 $(\mathrm{DBH}>22.5 \mathrm{~cm})$ 。

Fig. 2 Spatial distribution patterns of Picea schrenkiana with different ages (DBH) in the 8-ha forest dynamics plot in Tianshan Mountains. (A) Young tree $(1 \mathrm{~cm} \leqslant \mathrm{DBH} \leqslant 12.5 \mathrm{~cm})$; (B) Medium tree $(12.5 \mathrm{~cm}<\mathrm{DBH} \leqslant 22.5 \mathrm{~cm})$; (C) Mature tree (DBH $>$ $22.5 \mathrm{~cm})$.

增大，聚集程度减少(图3)。幼龄树和中龄树均在 $r=$ $1 \mathrm{~m}$ 时聚集强度最大(分别为3.91和1.98), 分别在 $r$ $\leqslant 8 \mathrm{~m}$ 和 $r \leqslant 10 \mathrm{~m}$ 时聚集强度下降幅度最大。老 龄树分布格局较复杂, 在 $0 \mathrm{~m} \leqslant r \leqslant 2 \mathrm{~m}$ 及 $r=4$ $\mathrm{m}$ 尺度上呈随机分布, 在其余尺度上均呈聚集分 布。总体来看, 雪岭云杉的聚集程度为: 幼龄树>中 龄树 $>$ 老龄树, 随着尺度的增加, 3 个径级的聚集强 度依次降低。

\section{3 密度制约效应}

利用随机标签零模型和案例-对照设计的方法 排除生境异质性后, 分析的雪岭云杉密度制约效应 结果见图4。以雪岭云杉幼龄树和中龄树作为案例
时, 在 $0-40 \mathrm{~m}$ 的尺度上 $g_{21}-g_{22}<0$, 并且位于包迹 线下方, 显示出幼龄树和中龄树相对于老龄树有额 外的聚集强度, 也即表现出密度制约效应。老龄树 $d(\mathrm{r})>0$, 并且密度制约效应强度介于 $0-2$ 之间(图 $4 \mathrm{C})$; 老龄树聚集程度的减幅在 $10 \mathrm{~m}$ 的小尺度内最 大，并随着尺度的增大而下降(图3C)。

\section{4 不同龄级雪岭云杉个体的空间关联}

基于随机标签零模型的双变量 $g$ 函数分析结果 (图5)表明，幼龄树和老龄树、中龄树和老龄树的个 体在 0-40 m的尺度上均呈现空间负关联, 且老龄树 与中龄树之间的负关联强度要比老龄树与幼龄树 之间的负关联强度小。 
A

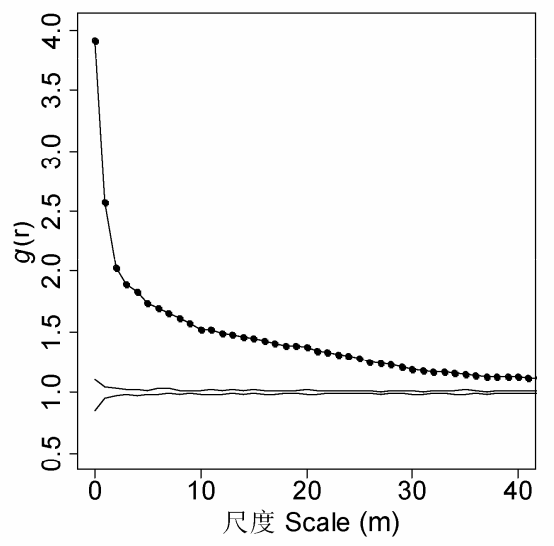

B

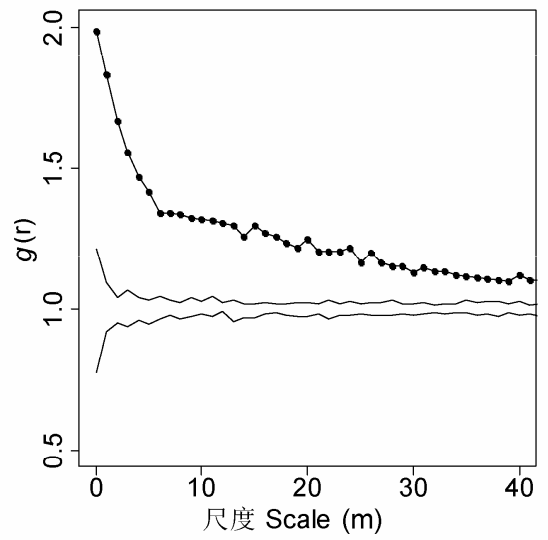

C

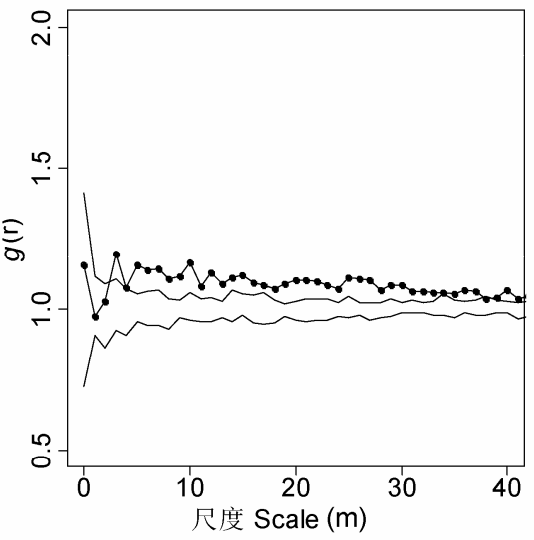

图3 天山雪岭云杉 $8 \mathrm{ha}$ 森林动态监测样地内不同龄级(径级)雪岭云杉个体空间分布格局的尺度依赖性。(A)幼龄树 $(1 \mathrm{~cm} \leqslant$ $\mathrm{DBH} \leqslant 12.5 \mathrm{~cm})$; (B)中龄树 $(12.5 \mathrm{~cm}<\mathrm{DBH} \leqslant 22.5 \mathrm{~cm})$; (C)老龄树 $(\mathrm{DBH}>22.5 \mathrm{~cm})$ 。实线为 $99 \%$ 置信区间的包迹线，点线 为实际点格局。

Fig. 3 Scale dependency of spatial patterns of Picea schrenkiana with different ages (DBH) in the 8-ha forest dynamics plot in Tianshan Mountains. (A) Young trees $(1 \mathrm{~cm} \leqslant \mathrm{DBH} \leqslant 12.5 \mathrm{~cm})$; (B) Medium trees $(12.5 \mathrm{~cm}<\mathrm{DBH} \leqslant 22.5 \mathrm{~cm})$; (C) Mature trees $(\mathrm{DBH}>22.5 \mathrm{~cm})$. Solid lines show 99\% simulation envelopes and solid lines with points represent the point pattern.

A

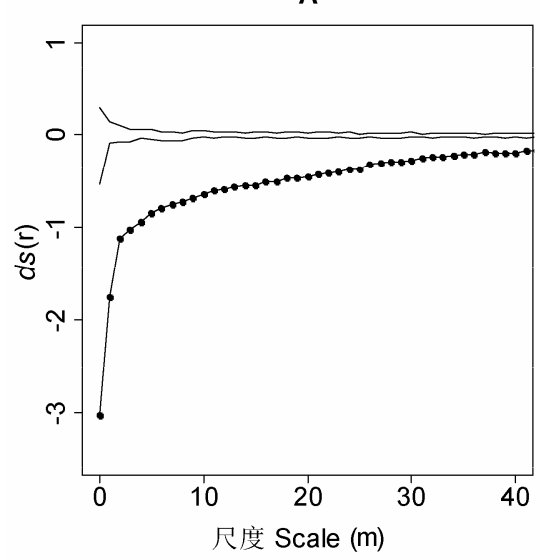

B

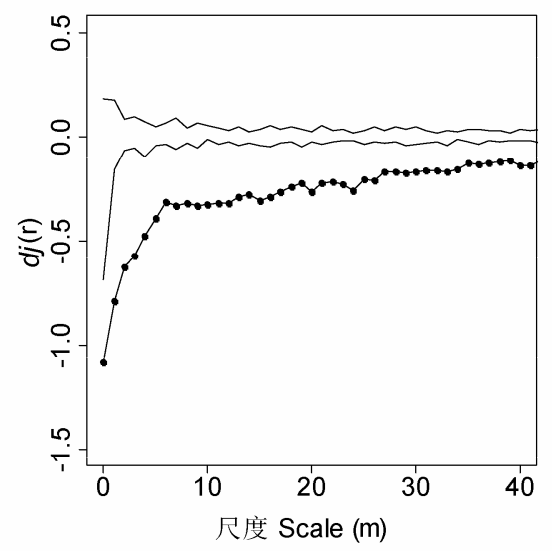

C

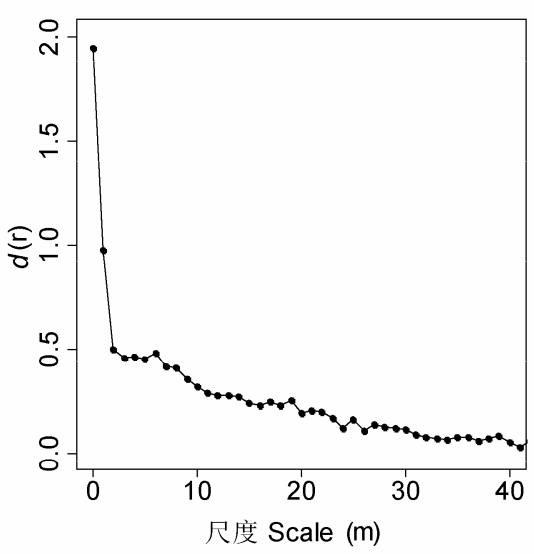

图4 天山雪岭云杉 8 ha森林动态监测样地密度制约效应分析。(A)幼龄树 $(1 \mathrm{~cm} \leqslant \mathrm{DBH} \leqslant 12.5 \mathrm{~cm})$ 作为案例, 老龄树(DBH $>22.5 \mathrm{~cm}$ )作为对照; (B)中龄树 $(12.5 \mathrm{~cm}<\mathrm{DBH} \leqslant 22.5 \mathrm{~cm}$ ) 作为案例, 老龄树作为对照; (C)从幼龄树到中龄树聚集格局的变 化。实线为 $99 \%$ 置信区间的包迹线, 点线为实际点的格局。

Fig. 4 Density-dependence analyses of the 8-ha Picea schrenkiana forest dynamics plot in Tianshan Mountains. (A) Young trees (1 $\mathrm{cm} \leqslant \mathrm{DBH} \leqslant 12.5 \mathrm{~cm})$ serve as case and mature trees $(\mathrm{DBH}>22.5 \mathrm{~cm})$ as control; (B) Medium trees $(12.5 \mathrm{~cm}<\mathrm{DBH} \leqslant 22.5$ $\mathrm{cm})$ serve as case and mature trees as control; (C) The pattern changes from young trees to medium trees. Solid lines show $99 \%$ simulation envelopes and solid lines with points represents the point pattern.

\section{3 讨论}

\section{1 样地内物种个体数量的动态变化}

在2009-2014年的5年间，天山雪岭云杉 8 ha森 林动态监测样地内 $\mathrm{DBH} \geqslant 1 \mathrm{~cm}$ 的个体死亡率为 $8.82 \%$, 补员率为 $2.19 \%$ 。以往研究中, 巴拿马 $\mathrm{BCI}$ (Barro Colorado Island)样地所有DBH $\geqslant 1 \mathrm{~cm}$ 的个 体 5 年间的死亡率为 $13.2 \%$ 、补员率为 $14.35 \%$
(Condit et al，1999); 长白山次生杨桦林5年间的死 亡率为 $13 \%$ 、补员率为 $4.05 \%$ (张昭臣等, 2013); 古 田山 5 ha样地所有个体的死亡率为 $10.1 \%$ 、补员率为 $33.25 \%$ (汪殷华等, 2011)。可见天山雪岭云杉 8 ha森 林动态监测样地的总体死亡率 $(8.82 \%)$ 和补员率 $(2.19 \%)$ 处于相对较低的水平, 与百山祖中山中亚 热带常绿阔叶林的情况(陈小荣等, 2013)接近。

物种的死亡率受多种因素影响, 一般来说个体 
A

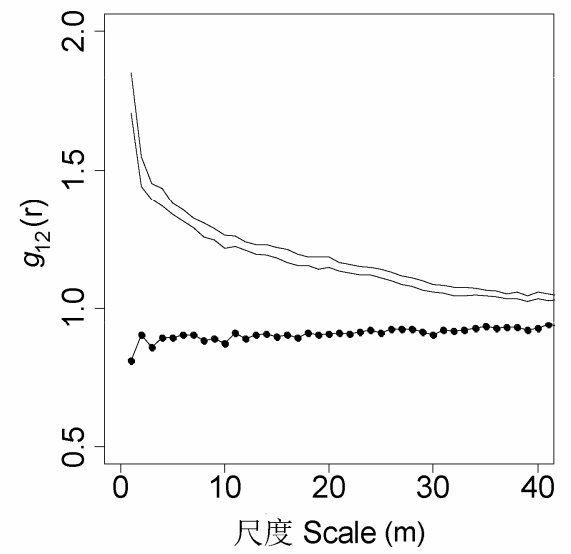

B

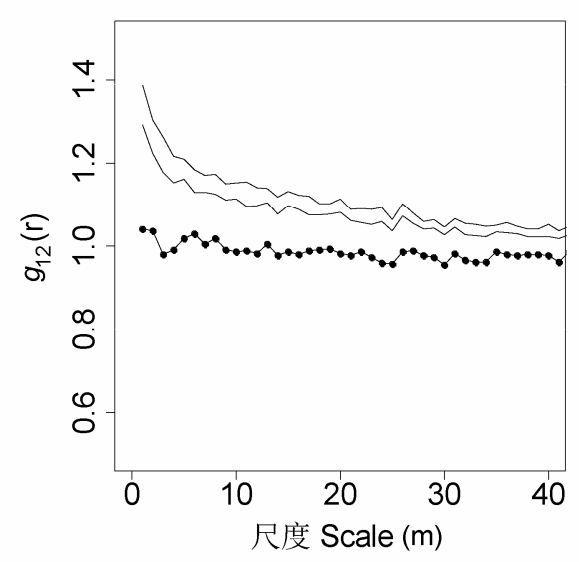

图5 天山雪岭云杉 $8 \mathrm{ha}$ 森林动态监测样地内不同龄级(径级)雪岭云杉的空间关联性。(A)幼龄树 $(1 \mathrm{~cm} \leqslant \mathrm{DBH} \leqslant 12.5 \mathrm{~cm})$ 与老龄树 $(\mathrm{DBH}>22.5 \mathrm{~cm})$; (B)中龄树 $(12.5 \mathrm{~cm}<\mathrm{DBH} \leqslant 22.5 \mathrm{~cm})$ 与老龄树。实线为 $99 \%$ 置信区间的包迹线, 点线为实际点的 格局。

Fig. 5 Spatial association of Picea schrenkiana with different ages (DBH) in the 8-ha forest dynamics plot in Tianshan Mountains. (A) Young $(1 \mathrm{~cm} \leqslant \mathrm{DBH} \leqslant 12.5 \mathrm{~cm})$ and mature $(\mathrm{DBH}>22.5 \mathrm{~cm})$ trees; (B) Medium $(12.5 \mathrm{~cm}<\mathrm{DBH} \leqslant 22.5 \mathrm{~cm})$ and mature trees. Solid lines show $99 \%$ simulation envelopes and solid lines with points represents the point pattern.

死亡率与其径级有关, 径级较小的个体容易死亡 (汪殷华等, 2011; 王斌等, 2014)。补员个体形成之 前, 还包括种子扩散、萌发及幼苗定植、生长等过 程。这些过程都可能对物种补员造成影响, 进而成 为限制植物种群更新的重要瓶颈(Clark et al, 1999)。

天山雪岭云杉林的林下缺乏可供更新的幼苗。 一方面可能是由于土层薄、水分条件相对较差、林 内光照不足不利于种子萌发, 从而导致了幼苗的匮 乏; 另一方面则可能是由于雪岭云杉叶和调落物中 存在3,4-二羟基苯乙酮等自毒物质, 抑制了其种子 的发芽和幼苗的生长, 从而导致群落更新出现障碍 (潘存德等, 2009)。幼苗溃乏是导致雪岭云杉森林较 低补员率的重要原因。

\section{2 雪岭云杉空间分布格局}

优势物种对群落结构的形成和维持有重要意 义。研究优势物种的空间结构有助于我们理解形成 群落中优势物种空间结构的生态过程, 以及其对其 他物种共存或群落结构维持的意义(He \& Duncan, 2000; 张雷等, 2011)。

种群的发育过程直接影响其个体的空间分布 格局, 不同发育阶段的个体往往处于不同林层, 表 现出不同的空间分布格局(王绪高等, 2008)。在本研 究中, 雪岭云杉不同发育阶段个体的空间分布格局 表现出一定的差异, 幼龄树和中龄树在 $r \leqslant 40 \mathrm{~m}$ 的尺度上表现为聚集分布, 聚集强度随着径级的增
大而减小, 然而老龄树却在小尺度上呈现随机分布 的趋势。究其原因, 可能是由于植物种群空间分布 格局是植物自身的生物学特性与环境因素共同作 用的结果(Harms et al, 2000; Queenborough et al, 2007; Lin et al, 2011)。雪岭云杉由于果实体积大、 质量重, 种子成熟后借助动物的搬运所能传播的距 离有限, 多数散落在母体周围的区域内, 这种生物 学特性使其在空间上易形成聚集分布的特征。然而 随着种群龄级的增大, 个体对光照、水分、营养和 空间等的需求增加, 种内和种间相互作用增强而造 成自疏或他疏作用, 导致种群的聚集强度逐渐减 弱, 种群由聚集分布向随机分布或均匀分布转变。

种内联结用来表示同一物种不同龄级在空间 分布上的相互关联性, 是对一定时期内植物种群组 成个体之间相互关系的静态描述。这种关系不仅包 括空间分布关系，也包含着个体间的功能关系，揭 示了种群的现状, 并能反映种群内个体间的相互作 用(王否等, 2010)。Huston和DeAngelis (1994)指出, 植物和土壤资源的相互作用及土壤中营养元素移 动的限制性将会造成各植物个体周围资源环境条 件的差异, 共存的植物一般在对不同资源的利用能 力上存在某种负相关关系。本研究表明, 随着龄级 差距的加大, 雪岭云杉幼龄树与老龄树、中龄树与 老龄树在 0-40 m尺度上均呈现明显的空间负关联, 说明幼龄树和中龄树受到老龄树的排斥。这主要是 
由于幼龄树与老龄树相比, 对土壤水分、光等资源 的竞争力微弱, 但它们对土壤养分和空间资源的要 求相同, 这种共存必然存在比较强烈的竞争。

\section{3 雪岭云杉的密度制约效应}

在天然林里, 种群在由幼龄树到老龄树发展的 过程中, 聚集程度通常会逐渐降低, 一般趋势为: 聚集 $\rightarrow$ 随机 $\rightarrow$ 均匀(Coomes et al, 2003)。这种格局的 变化源于群落中特定机制的影响, 生境异质性可能 是影响种群格局发生变化的重要因子。有研究发现, 排除生境异质性后, 密度制约是调节种群结构的重 要机制(Zhu et al, 2010)。同种个体通过密度制约效 应减少了个体数量, 为其他物种入侵和定居提供了 空间, 从而实现物种共存。

本文结果显示, 在剔除了生境异质性干扰后, 雪岭云杉幼龄树和中龄树相对于老龄树有额外的 聚集现象, 即表现出密度制约效应。相对于老龄树, 幼龄树聚集程度的减幅一般在 $10 \mathrm{~m}$ 的小尺度内最 大, 这可能是由于物种专一性的资源分布不均, 导 致老龄树与非老龄树之间的非对称竞争(Getzin et al, 2008)。在林下个体生长过程中, 密集的非老龄树 充分利用林下空间，同时种内竞争加剧(祝燕等, 2011)，造成大量非老龄树死亡，使雪岭云杉非老龄 树相对于老龄树产生的额外聚集程度减弱。这与其 他学者对不同物种密度制约效应的研究结果一致 (Zhu et al, 2010; 张受等, 2014)。

\section{参考文献}

Aminem E, Chang SL, Zhang YT, Qiu Y, He P (2014) Altitudinal distribution rule of Picea schrenkiana forest's soil organic carbon and its influencing factors. Acta Ecologica Sinica, 34, 1626-1634. (in Chinese with English abstract) [阿米娜木-艾力, 常顺利, 张毓涛, 仇瑶, 何平(2014) 天 山云杉森林土壤有机碳沿海拔的分布规律及其影响因素. 生态学报, 34, 1626-1634.]

Bagchi R, Henrys PA, Brown PE, Burslem DFRP, Diggle PJ, Savitri GCV, Nimal GIAU, Kassim AR, Law R, Noor S, Valencia RL (2011) Spatial patterns reveal negative density dependence and habitat associations in tropical trees. Ecology, 92, 1723-1729.

Chen XR, Chen YY, Luo ZR, Ding BY (2013) A 5-year mid-mountain subtropical evergreen broad-leaved forest study in Baishanzu, East China. Journal of Zhejiang A \& F University, 30, 821-829. (in Chinese with English abstract) [陈小荣, 陈圆圆, 骆争荣, 丁炳扬 (2013) 百山祖中山中 亚热带常绿阔叶林群落 5 年动态特征. 浙江农林大学学 报, 30, 821-829.]

Clark JS, Silman M, Kern R, Macklin E, Hille RJ (1999) Seed dispersal near and far: patterns across temperate and tropical forests. Ecology, 80, 1475-1494.

Connell JH (1971) On the role of natural enemies in preventing competitive exclusion in some marine animals and in rain forest trees. In: Dynamic of Populations (eds den Boer PJ, Gradwell G), pp. 298-312. Center Ag Publishing \& Documentation Wageningen, Wageningen,.

Chen L, Mi XC, Comita L, Zhang LW, Ren HB, Ma KP (2010) Community-level consequences of density dependence and habitat association in a subtropical broad-leaved forest. Ecology Letters, 13, 695-704.

Condit R (1998) Ecological implications of changes in drought patterns: shifts in forest composition in Panama. Climatic Change, 39, 413-427

Condit R, Ashton PS, Baker P, Bunyavejchewin S, Gunatilleke S, Gunatilleke N, Hubbel SP, Foster RB, Itoh A, LaFrankie JV, Lee HS, Losos E, Manokaran N, Sukumar R, Yanakura $\mathrm{T}$ (2000) Spatial patterns in the distribution of tropical tree species. Science, 288, 1414-1418.

Condit R, Ashton PS, Manokaran N, LaFrankie JV, Hubbell SP, Foster RB (1999) Dynamics of the forest communities at Pasoh and Barro Colorado: comparing two 50-ha plots. Philosophical Transactions of the Royal Society of London B: Biological Sciences, 354, 1739-1748.

Coomes DA, Duncan RP, Allen RB, Truscott J (2003) Disturbances prevent stem size-density distributions in natural forests from following scaling relationships. Ecology Letters, 6, 980-989.

Diggle PJ, Chetwynd AG (1991) Second order analysis of spatial clustering for inhomogeneous populations. Biometrics, $47,1155-1163$.

Getzin S, Wiegand T, Wiegand K, He FL (2008) Heterogeneity influences spatial patterns and demographics in forest stands. Journal of Ecology, 96, 807-820.

Gunton RM, Kunin WE (2009) Density-dependence at multiple scales in experimental and natural plant populations. Journal of Ecology, 97, 567-580.

Guo YL, Lu ZJ, Wang QG, Lu JM, Xu YZ, Meng HJ, Liu HB, Zhang JX, Bao DC, Qiao XJ, Huang HD, Jiang MX (2015) Detecting density dependence from spatial patterns in a heterogeneous subtropical forest of Central China. Canadian Journal of Forest Research, 45, 710-720

Guo YL, Wang B, Xiang WS, Ding T, Lu SH, Huang FZ, Li DX, Wen SJ, He YL, Li XK (2015) Dynamics of density-dependent effects of tree species in a 15 ha seasonal rain forest plot in northern tropical karst in Nonggang, Guangxi, southern China. Chinese Science Bulletin, 60, 1602-1611. (in Chinese with English abstract) [郭屹立, 王斌, 向悟生, 丁涛, 陆树华, 黄甫昭, 李冬兴, 文淑均, 何运林, 李先 琨 (2015)弄岗喀斯特季节性雨林15 ha样地密度制约效应 分析. 科学通报, 60, 1602-1611.]

Harms KE, Wright SJ, Calderon O, Hernandez A, Herre EA (2000) Pervasive density-dependent recruitment enhances seedling diversity in a tropical forest. Nature, 404, 493-495.

He F, Duncan RP (2000) Density-dependent effects on tree survival in an old-growth Douglas fir forest. Journal of 
Ecology, 88, 676-688.

Huston MA, DeAngelis DL (1994) Competition and coexistence: the effects of resource transport and supply rates. The American Naturalist, 144, 954- 977.

Hubbell SP, Ahumada JA, Condit R, Foster RB (2001) Local neighborhood effects on long-term survival of individualtrees in a Neotropical forest. Ecological Research, 16, 859875.

Janzen DH (1970) Herbivores and the number of tree species in tropical forests. The American Naturalist, 104, 501-528.

Li L, Chen JH, Ren HB, Mi XC, Yu MJ, Yang B (2010) Spatial patterns of Castanopsis eyrei and Schima superba in mid-subtropical broad-leaved evergreen forest in Gutianshan National Nature Reserve, China. Chinese Journal of Plant Ecology, 34, 241-252. (in Chinese with English abstract) [李立，陈建华，任海保，米湘成，于明坚，杨波 (2010) 古田山常绿阔叶林优势树种甜槠和木荷的空间格局分析. 植物生态学报, 34, 241-252.]

Lin YC, Chang LW, Yang KC, Wang HH, Sun IF (2011) Point patterns of tree distribution determined by habitat heterogeneity and dispersal limitation. Oecologia, 165, 175-184.

Luo ZR (2011) The Effects of Density Dependence in Subtropical Evergreen Broad-leaved Forest of Baishanzu. PhD dissertation, Zhejiang University, Hangzhou. (in Chinese with English abstract) [骆争荣 (2011) 百山祖亚热带常绿 阔叶林群落密度制约的作用. 博士学位论文, 浙江大学, 杭州.]

Luo ZR, Mi XC, Chen XR, Ye ZL, Ding BY (2012) Density dependence is not very prevalent in a heterogeneous subtropical forest. Oikos, 121, 1239-1250.

Ma KP (2013) Studies on biodiversity and ecosystem function via manipulation experiments. Biodiversity Science, 21 247-248. (in Chinese) [马克平 (2013) 生物多样性与生态 系统功能的实验研究. 生物多样性, 21, 247-248.]

Pan CD, Wang Q, Ruan X, Li ZH (2009) Biological activity and quantification of potential autotoxins from the leaves of Picea schtenkiana. Chinese Journal of Plant Ecology, 33, 186-196. (in Chinese with English abstract) [潘存德, 王强, 阮晓, 李兆慧 (2009) 天山云杉针叶水提取物自毒效应 及自毒物质的分离鉴定. 植物生态学报, 33, 186-196.]

Piao TF, Comita LS, Jin GZ, Kim JH (2013) Density dependence across multiple life stages in a temperate old-growth forest of Northeast China. Oecologia, 172, 207-217.

Qiu Y, Chang SL, Zhang YT, Wang WD, He P, Wang HJ, Xie J (2015) Biomass estimation modeling and adaptability analysis of organ allocation in six common shrub species in the forests of Tianshan Mountains. Acta Ecologica Sinica, 35, 7842-7851. (in Chinese with English abstract) [仇瑶, 常顺利, 张毓涛, 王文栋, 何平, 王慧杰, 谢锦 (2015) 天山林区六种灌木生物量的建模及其器官分配的适应性 分析. 生态学报, 35, 7842-7851.]

Queenborough SA, Burslem D, Garwood NC, Valencia R (2007) Habitat niche partitioning by 16 species of Myristicaceae in Amazonian Ecuador. Plant Ecology, 192, 193-
207.

Ripley BD (1977) Modelling spatial patterns. Journal of the Royal Statistical Society Series B: Statistical Methodology, 39, 172-212.

Stoll P, Newbery DM (2005) Evidence of species-specific neighborhood effects in the Dipterocarpaceae of a Bornean rain forest. Ecology, 86, 3048-3062.

Wang B, Huang YS, Li XK, Xiang WS, Ding T, Huang FZ, Lu SH, Han WH, Wen SJ, He LJ (2014) Species composition and spatial distribution of a 15 ha northern tropical karst seasonal rain forest dynamics study plot in Nonggang, Guangxi, southern China. Biodiversity Science, 22, 141156. (in Chinese with English abstract) [王斌, 黄俞淞, 李 先琨, 向悟生, 丁涛, 黄甫昭, 陆树华, 韩文衡, 文淑均, 何兰军 (2014) 弄岗北热带喀斯特季节性雨林15 ha监测 样地的树种组成与空间分布. 生物多样性, 22, 141-156.]

Wang BS (1987) Phytocoenology. Higher Education Press, Beijing. (in Chinese) [王伯荪 (1987). 植物群落学. 高等 教育出版社, 北京.]

Wang L, Sun QW, Hao CY, Tian SN, Zhang SS, Chen YK, Zhang XP (2010) Point pattern analysis of different age-class Taxus chinensis var. mairei individuals in mountainous area of southern Anhui Province. Chinese Journal of Applied Ecology, 21, 271-278. (in Chinese with English abstract) [王磊, 孙启武, 郝朝运, 田胜尼, 张姗姗, 陈一锟, 张小平 (2010) 皖南山区南方红豆杉种群不同龄级立木 的点格局分析. 应用生态学报, 21, 271-278.]

Wang T, Ren SY, Yuan ZL, Zhu Y, Pan NL, Lu X, Ye YZ (2014) Effects of density dependence on the spatial patterns of Quercus aliena var. acuteserrata trees in deciduous broad-leaved forest in the Baotianman National Nature Reserve, Central China. Biodiversity Science, 22, 449-457. (in Chinese with English abstract) [王婷, 任思远, 袁志良, 祝 燕, 潘娜李, 鹿金金, 叶永忠 (2014) 密度制约对宝天曼落 叶阔叶林锐齿栋死亡前后分布格局的影响. 生物多样性, 22, 449-457.]

Wang W, Rao MD, Chen SW, Zhu DH, Mi XC, Zhang JT (2014) Effects of negative density dependence and habitat filtering on temporal variation in phylogenetic community structure of seedlings in a mid-subtropical forest. Chinese Science Bulletin, 59, 1844-1850. (in Chinese with English abstract) [王薇, 饶米德, 陈声文, 朱大海, 米湘成, 张金 屯 (2014) 负密度制约和生境过滤对古田山幼苗系统发 育多样性时间变化的影响. 科学通报, 59, 1844-1850.]

Wang XG, Wiegand T, Wolf A, Howe R, Davies S, Hao ZQ (2011) Spatial patterns of tree species richness in two temperate forests. Journal of Ecology, 99, 1382-1393.

Wang XG, Hao ZQ, Ye J, Zhang J, Li BH, Yao XL (2008) Relationships between species abundance and spatial distribution pattern of broad-leaved Korean pine (Pinus koraiensis) mixed forest in Changbai Mountains of China. Chinese Journal of Ecology, 27, 145-150. (in Chinese with English abstract) [王绪高, 郝占庆, 叶吉, 张健, 李步杭, 姚晓琳 (2008) 长白山阔叶红松林物种多度和空间分布格局. 生 
态学杂志, 27, 145-150.]

Wang XT, Hou YL, Liang CZ, Wang W, Liu F (2012) Point pattern analysis based on different null models for detecting spatial patterns. Biodiversity Science, 20, 151-158. (in Chinese with English abstract) [王金錘厅, 侯亚丽, 梁存柱, 王 炜，刘芳 (2012) 基于不同零模型的点格局分析. 生物多 样性, 20, 151-158.]

Wang YH, Mi XC, Chen SW, Li MH, Yu MJ (2011) Regeneration dynamics of major tree species during 2002-2007 in a subtropical evergreen broad-leaved forest in Gutianshan National Nature Reserve in East China. Biodiversity Science, 19, 178-189. (in Chinese with English abstract) [汪殷 华, 米湘成, 陈声文, 李铭红, 于明坚 (2011) 古田山常 绿阔叶林主要树种2002-2007年间更新动态. 生物多样 性, 19, 178-189.]

Wiegand T, Moloney KA (2004) Rings, circles and nullmodels for point pattern analysis in ecology. Oikos, 104, 209-229.

Zhang J, Hao ZQ, Sun IF, Song B, Ye J, Li BH, Wang XG (2009) Density dependence on tree survival in an old-growth temperate forest in northeastern China. Annals of Forest Science, 66(2), 1-9.

Zhang JT (1995) Methods in Vegetation Quantitative Ecology. China Science and Technology Press, Beijing. (in Chinese) [张金屯 (1995) 植被数量生态学方法. 中国科学技术出 版社, 北京.]

Zhang L, Wang XJ, Hu EC, Gao GY, Pang X, Yu YZ, Zhang ZH (2011) Population structure and spatial pattern of Ulmus macrocarpa var. mongolica in Horqin Sandy Land, China. Journal of Desert Research, 31, 115-119. (in Chinese with English abstract) [张雷, 王晓江, 胡尔查, 高桂英, 庞金金, 于有忠, 张振华 (2011) 科尔沁沙地蒙古黄榆种群结构 与空间分布格局. 中国沙漠, 31, 115-119.]

Zhang M, Mi XC, Jin GZ (2014) Composition and spatial patterns of the Liangshui spruce-fir valley forest in the Xiao Hinggan Mountains. Chinese Science Bulletin, 59, 2377-2387. (in Chinese with English abstract) [张受, 米湘 成, 金光泽 (2014) 小兴安岭凉水谷地云冷杉林群落组 成与空间格局. 科学通报, 59, 2377-2387.]
Zhang YT, Chang SL, Lu JJ, Li X, Wang Z, Shi QD, Zhang XP (2011a) Large scale permanent plot developed and its three-dimension realized in Tianshan forest. Scientia Silvae sinicae, 47, 179-183. (in Chinese with English abstract) [张 毓涛, 常顺利, 芦建江, 李翔, 王智, 师庆东, 张新平 (2011a) 天山云杉森林 8 ha样地的建立及三维可视化管 理. 林业科学, 47, 179-183.]

Zhang YT, Li JM, Chang SL, Li X, Lu JJ (2011b) Spatial distribution pattern of Picea schrenkiana var. tianshanica population and its relationships with topographic factors in middle part of Tianshan Mountain. Chinese Journal of Applied Ecology, 22, 2799-2806. (in Chinese with English abstract) [张毓涛, 李吉玫, 常顺利, 李翔, 芦建江 (2011b) 天山中部天山云杉种群空间分布格局及其与地形因子的 关系. 应用生态学报, 22, 2799-2806.]

Zhang ZC, Hao ZQ, Ye J, Lin F, Yuan ZQ, Xing DL, Shi S, Wang XG (2013) Short-term death dynamics of trees in natural secondary poplar-birch forest in Changbai Mountains of Northeast China. Chinese Journal of Applied Ecology, 24, 303-310. (in Chinese with English abstract) [张昭 臣, 郝占庆, 叶吉, 萄菲, 原作强, 邢丁亮, 师帅, 王绪高 (2013) 长白山次生杨桦林树木短期死亡动态. 应用生态 学报, 24, 303-310.]

Zhu Y, Mi XC, Ma KP (2009) A mechanism of plant species coexistence: the negative density-dependent hypothesis. Biodiversity Science, 17, 594-604. (in Chinese with English abstract) [祝燕, 米湘成, 马克平 (2009) 植物群落物种共 存机制: 负密度制约假说. 生物多样性, 17, 594-604.]

Zhu Y, Bai F, Liu HF, Li WC, Li L, Li GQ, Wang SZ, Sang WG (2011) Population distribution patterns and interspecific spatial associations in warm temperate secondary forests, Beijing. Biodiversity Science, 19, 252-259. (in Chinese with English abstract) [祝燕, 白帆, 刘海丰, 李文超, 李亮, 李 广起, 王顺忠, 桑卫国 (2011) 北京暖温带次生林种群分 布格局与种间空间关联性. 生物多样性, 19, 252-259.]

Zhu Y, Mi XC, Ren HB, Ma KP (2010) Density dependence is prevalent in a heterogeneous subtropical forest. Oikos, 119, 109-119. 